\title{
Multifeature Detection of Microaneurysms Based on Improved SSA
}

\author{
Liwei Deng ${ }^{1}\left(\mathbb{D}\right.$, Xiaofei Wang ${ }^{2, *} \mathbb{D}$ and Jiazhong $\mathrm{Xu} \mathrm{u}^{1,2} \mathbb{D}$ \\ 1 Heilongiiang Provincial Key Laboratory of Complex Intelligent System and Integration, \\ School of Automation, Harbin University of Science and Technology, Harbin 150080, China; \\ dengliwei666@hrbust.edu.cn (L.D.); xujiazhong@hrbust.edu.cn (J.X.) \\ 2 Key Laboratory of Advanced Manufacturing and Intelligent Technology Ministry of Education, \\ Harbin University of Science and Technology, Harbin 150080, China \\ * Correspondence: 1820510049@stu.hrbust.edu.cn; Tel.: +86-045186390863
}

Citation: Deng, L.; Wang, X.; Xu, J. Multifeature Detection of Microaneurysms Based on Improved SSA. Symmetry 2021, 13, 2147. https://doi.org/10.3390/sym13112147

Academic Editors: Qinghua Huang and Haigang Ma

Received: 8 October 2021

Accepted: 1 November 2021

Published: 10 November 2021

Publisher's Note: MDPI stays neutral with regard to jurisdictional claims in published maps and institutional affiliations.

Copyright: (c) 2021 by the authors. Licensee MDPI, Basel, Switzerland. This article is an open access article distributed under the terms and conditions of the Creative Commons Attribution (CC BY) license (https:// creativecommons.org/licenses/by/ $4.0 /)$.

\begin{abstract}
The early diagnosis of retinopathy is crucial to the prevention and treatment of diabetic retinopathy. The low proportion of positive cases in the asymmetric microaneurysm detection problem causes preprocessing to treat microaneurysms as noise to be eliminated. To obtain a binary image containing microaneurysms, the object was segmented by a symmetry algorithm, which is a combination of the connected components and SSA methods. Next, a candidate microaneurysm set was extracted by multifeature clustering of binary images. Finally, the candidate microaneurysms were mapped to the Radon frequency domain to achieve microaneurysm detection. In order to verify the feasibility of the algorithm, a comparative experiment was conducted on the combination of the connected components and SSA methods. In addition, PSNR, FSIM, SSIM, fitness value, average CPU time and other indicators were used as evaluation standards. The results showed that the overall performance of the binary image obtained by the algorithm was the best. Last but not least, the accuracy of the detection method for microaneurysms in this paper reached up to $93.24 \%$, which was better than that of several classic microaneurysm detection methods in the same period.
\end{abstract}

Keywords: SSA; connection components; diabetic retina; asymmetric microaneurysm; Radon transform

\section{Introduction}

Diabetic retinopathy (DR) is one of the four leading causes of blindness in the world; it causes blindness mainly in working-age adults around the world [1]. The other three leading causes are cataract, glaucoma and maculopathy [2]. A global assessment of the prevalence of DR in a 2012 study, which used data from population-based studies worldwide, found that DR patients accounted for $34.6 \%$ of diabetics [3]. Previous studies have demonstrated significant differences between diagnosed and undiagnosed DR in prevalence. An estimated 100 million people in China and 80 million people in India have diabetes $[4,5]$. This means that the early diagnosis of DR is important. The early lesions of DR mainly include microaneurysms (MAs) and rigid exudations, among others. DR is clinically diagnosed with several lesions (MA, hard exudate and soft exudate) at the initial stages [6]. Some studies have shown that the presence of even one or two dynamic MAs increases the rate of accelerated disease progression [7,8]. Moreover, the number of MAs is significantly different among different graders, but the identification of MAs is relatively consistent after image processing [9]. Common fundus diagnostic methods include RGB fundus angiography and fundus fluorescein angiography (FFA). In this paper, the research object was FFA, because FFA images have a unique role in the diagnosis of fundus diseases, especially for the diagnosis of vascular diseases (MA is a disease that acts on fundus blood vessels) [10]. MA is a kind of fundus oculi lesion that can be accurately diagnosed using FFA [11]. FFA is an angiographic method in which sodium fluorescein is injected into the patient's blood vessels to make the angiography more visible and more easily differentiated from surrounding tissue. FFA was first proposed by Novotny and Alvis in 1961, and it 
has since contributed to the development of MA detection [12]. Aiming to achieve high contrast of MAs in FFA images, Quan Qi segmented the MAs in FFA images by OTSU [13]. Manual segmentation of fundus images is low in efficiency, costly and difficult to perform. Many scholars introduced metaheuristic algorithms for multiple optimal threshold values of fitness functions to solve the problem mentioned above. In order to improve the segmentation speed, fundus images were segmented by whale optimization algorithm (WOA), genetic algorithm (GA) and ant colony optimization (ACO) algorithm [14-16]. The above metaheuristic algorithms (in the literature [14-16]) effectively explore from global thresholds to local thresholds. Metaheuristic algorithms are used widely as evolutionary algorithms and swarm intelligence optimization algorithms [17]. Intelligent optimization algorithms have excellent performance in the field of image processing, but there is no perfect algorithm to solve all optimization problems [18]. Considering the engineering perspective, a faster and more accurate multithreshold image segmentation method is urgently needed. In this paper, DR FFA images were segmented by the salp swarm algorithm (SSA) technique, an algorithm initially proposed by Mirjalili et al. [19]. The SSA has advantages in real-time medical image processing because it iterates quickly and easily converges and obtains the global optimal solution. The biggest problem of common population optimization algorithms is that they can easily fall into local optimization. An advantage of the SSA in regard to this problem is that the exploration and exploitation ratio of the algorithm is adjustable, which makes it possible to change update by adjusting the exploration and exploitation ratio. Literally, threshold segmentation is the clustering of pixels according to the threshold value $T$. Threshold techniques fall into two categories: global threshold methods and local threshold methods. In the former, a common threshold is shared by all pixels of the image [20]. In the latter, a uniform threshold is shared by all pixels in a region separated from the image according to certain rules. In many cases, the two methods are combined to segment an image [21]. Local techniques are commonly used for complex inputs and irregular lighting and noise distribution. The local threshold method is faster than the global method, but it is not suitable for complex inputs as described above. Therefore, we extended the research field to multiple dimensions. In order to verify the performance of the connected components with SSA (CSSA) for the segmentation of fundus images, a comparative experiment was performed with extreme optimization (EO), simplified swarm optimization (SSO), WOA, particle swarm optimization (PSO) and GA. The experimental results showed that the binary image created by CSSA contained more information, which contributed to the accuracy of subsequent MA detection [22-26]. MA testing is divided into fully supervised learning and semisupervised learning (SSL). The field of fully supervised learning for MA positioning has grown rapidly in recent years. However, because of the limitations of FFA image datasets, fully supervised learning is applied mainly to MA localization of color fundus images. Norah Asir proposed a dual-stream red lesion detection system based on faster RCNN for MA detection [27]. Cheng Wan proposed EAD-Net, incorporating the attention mechanism for MA detection [28]. SSL is a learning method that combines supervised learning with unsupervised learning. SSL uses a large amount of unlabeled data. It is increasingly important because it requires fewer people to do the work while delivering higher accuracy compared to fully supervised learning. Because FFA has few public datasets, many scholars have used SSL. Gwénolé Quellec located MAs by matching the subbands of a wavelet transform image and a mathematical model of MAs [29]. In the literature [30], generative adversarial networks and thermal regression have been used to implement multifeature detection for MA. Marwan D. Saleh segmented h-maxima transformed images with multiple features to achieve MA detection [31].

The main content of this paper is as follows: First, to obtain an asymmetric binary image containing MAs, an FFA image was multidimensionally segmented by combining the connected components with the CSSA. Second, the binary images were multifeature clustered by the MSA-K algorithm to obtain a set of candidate MAs. Third, a preprocessing operation was performed on the candidate MA windows extracted from the original image. Finally, the windows were mapped to the Radon domain with multiple 
angles $\theta_{\mathrm{i}} \quad(\mathrm{i}=1,2, \cdots, \mathrm{m})$ to obtain $\operatorname{Radon}\left(M A-\right.$ window $\left._{\mathrm{j}}\right)$, and the detection of MA was achieved by clustering the standard deviation values between the central band of $\operatorname{Radon}\left(M A-\right.$ window $\left._{\mathrm{j}}\right)$.

The method of this paper can be implemented on a simple and basic operating platform, which means that the algorithm has low requirements on the operating environment and low economic costs. The multithreshold processing of diabetic retinal images by CSSA has greatly reduced the workload of doctors and reduced the cost of diabetes diagnosis.

\section{Materials and Methods}

This paper did not involve human-related experiments. All the pictures in the simulation section were obtained from the Internet.

As shown in Figure 1, the asymmetric MA detection in this paper included binary images, sets of candidate MAs and locations of MAs. MAs were highlighted as dots in FFA images, as shown in Figure 2 [32].

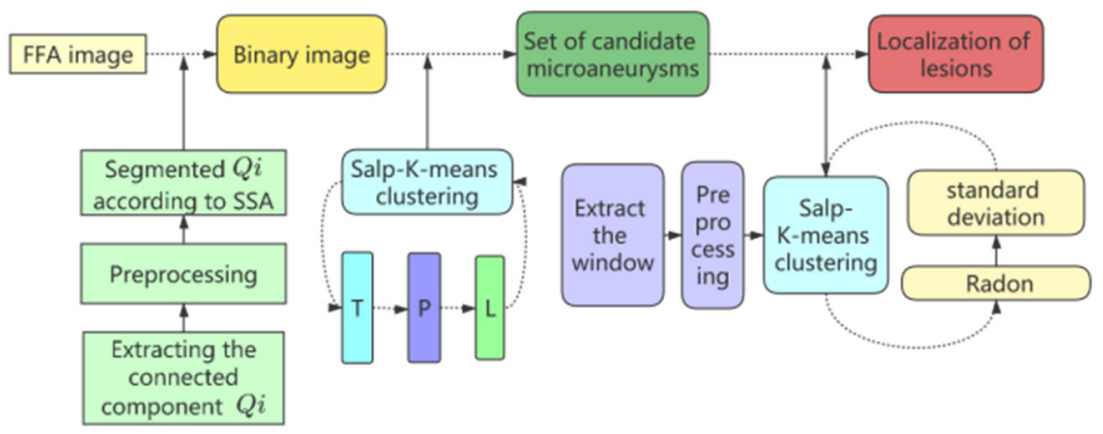

Figure 1. Block diagram of asymmetric MA detection.



Figure 2. Enlarged view of an MA.

\subsection{Extraction of Binary Images}

2.1.1. SSA

The SSA is a new swarm optimization algorithm that simulates salp foraging behavior in the deep ocean. The mathematical model of the salp chain includes leaders and followers. The former contributes to exploration and the latter contributes to exploitation. The fronts of salp chains are defined as leaders, and the salps for the rest of the chain are defined as followers [19]. As this sentence implies, leaders lead the salp chain forward and explore the search space, and followers lead each other. A salp chain is shown in Figure 3. 


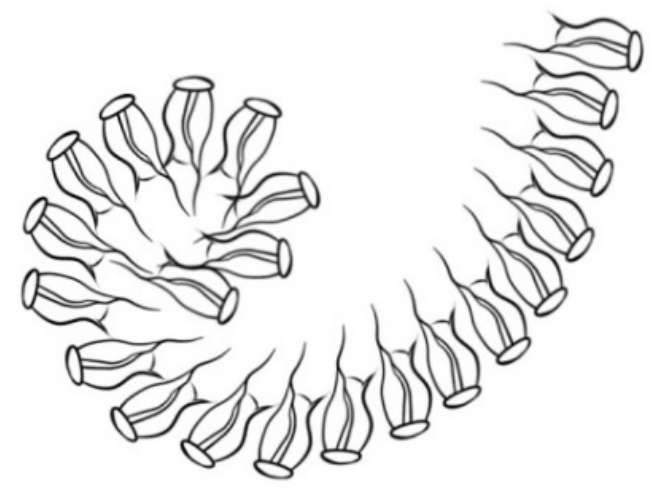

Figure 3. Salp chain.

The SSA is a stochastic algorithm to avoid falling into local optima stagnation. The weights of SSA exploration and exploitation can be changed by changing the proportion of leaders and followers. As in other swarm algorithms, elements in the SSA explore the $\mathrm{N}$-dimensional search space. It is assumed that two-dimensional matrixes store the position of the salp, called X, and that of the swarm's target in the search space, represented by the food source, called $F$.

The equation for the position update is as follows:

$$
\begin{array}{cc}
x_{n}^{i}=F_{n}+c_{1} \times\left(\left(\mathrm{ub}_{n}-l \mathrm{~b}_{n}\right) \times \mathrm{c}_{2}+l \mathrm{~b}_{n}\right) & c_{3} \geq 0.5 \\
x_{n}^{i}=F_{n}-c_{1} \times\left(\left(\mathrm{ub}_{n}-l \mathrm{~b}_{n}\right) \times \mathrm{c}_{2}+l \mathrm{~b}_{n}\right) & c_{3}<0.5 \\
x_{n}^{i}=\frac{1}{2} x_{n}^{i}-x_{n}^{i-1} &
\end{array}
$$

where $X_{n}^{i}$ is the position of the $i$ th salp follower, $N$ is the number of variables covered with the objective problem, $F_{n}$ is the position of the food for the $n$th variable and $l b_{n}$ and $u b_{n}$ indicate the lower and upper limitations of the $n$th variable, respectively. The parameter $c_{1}$ balances exploration and exploitation by changing the ratio of leaders to followers as follows:

$$
c_{1}=2 e^{-\left(\frac{4 L}{l}\right)^{2}}
$$

where $l$ is the current iteration number of the algorithm, $L$ is the total iteration number of the algorithm and $c_{3}$ determines the forward direction of the chain. When $c_{3} \geq 0.5$, the chain moves towards $+\infty$, and when $c_{3}<0.5$, the chain moves towards $-\infty . c_{2}$ determines the length of the chain's movement. A schematic diagram of a salp chain update is shown in Figure 4.

The initial salp position in the SSA is random, which means that the SSA can explore a problem in multiple directions. The positions of leaders are updated according to Equations (1) and (2), and the positions of followers are updated according to Equation (3). The SSA is better at exploration than most ACO-like swarm algorithms because the position of its followers is determined only by that of the previous salp. In addition, the location of leaders depends on the food $F_{n} . F_{n}$ is the position of the salp with the best fitness value in the current iteration $l$. The higher the percentage of leaders, the more random the chain is. In this paper, 30 salps were selected, among which the first 15 were leaders and the last 15 were followers, to avoid falling into local optimal solutions. 

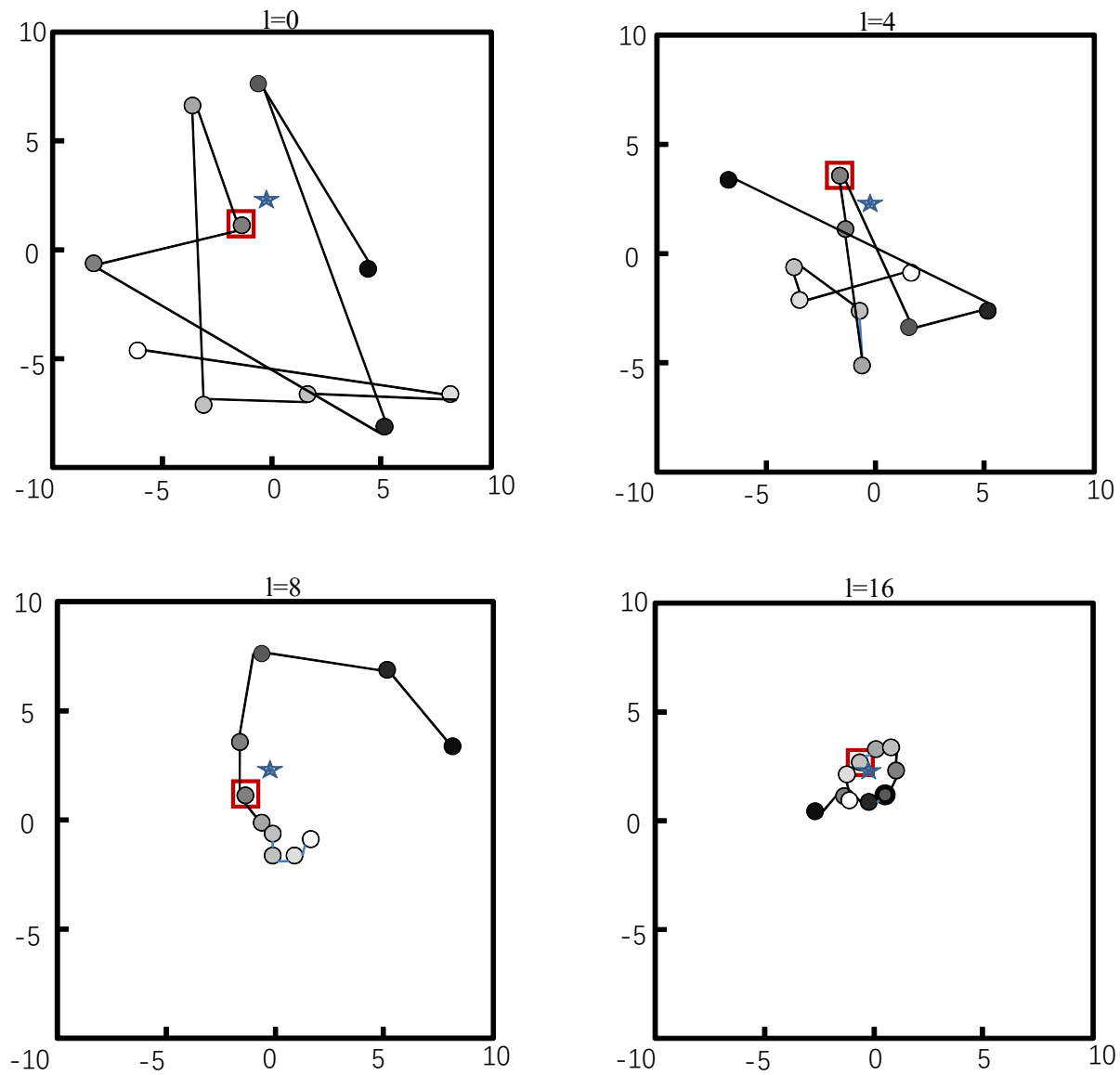

Figure 4. Schematic diagram of a salp chain searching for optimal solutions in two dimensions. $\hbar$ is the optimal solution; $\square$ is the swarm's optimal $F_{n}$ for the current iteration $l$.

\subsubsection{CSSA}

The segmentation threshold of globally connected components depends on the global probability of pixel distribution. In general, global threshold segmentation results in the loss of image details. Experiments have shown that the lesions in DR images are extracted by global threshold methods in a distorted manner. In particular, some blood vessel endings became broken blood vessels under global threshold methods. In order to solve this problem, symmetry CSSA is proposed herein. Each connected component identified by global threshold methods is subdivided into several subregions, different thresholds are calculated for different subregions and subregions are processed according to local thresholds. The SSA is used to segment subgraphs based on multiple thresholds. In this paper, we defined the number of individuals in the salp group $\mathrm{K}=30$, among which the first 15 were defined as leaders and the last 15 were defined as followers. In order to transform the image threshold segmentation problem into a multidimensional solution problem, OTSU was selected as the fitness function of the SSA.

The OTSU algorithm is an efficient image segmentation method for binary images [33]. It assumes that an image, with size $\mathrm{M} \times \mathrm{N}$ and gray levels belonging to $[0,1, \ldots, \mathrm{L}-1]$, is divided into two parts (foreground and background) with level T [34].

The variance between classes is:

$$
\delta_{B}^{2}(\mathbf{t})=\omega_{0}\left(\mu_{0}-\mu_{1}\right)^{2}+\omega_{1}\left(\mu_{1}-\mu_{1}\right)^{2}
$$

For CSSA, the first step is the extraction of connected components. An FFA image $f(m, n)$ includes the foreground (object region) and background, and the gray value of the pixels belonging to these two ranges is significantly different [35]. 
Threshold processing can be treated as a function:

$$
T=T[\mathrm{~m}, \mathrm{n}, p(\mathrm{~m}, \mathrm{n}), f(\mathrm{~m}, \mathrm{n})]
$$

where $p(\mathrm{~m}, \mathrm{n})$ is the characteristic of the points distributed around the point $(\mathrm{m}, \mathrm{n})$ and $f(m, n)$ is the gray level of point $(m, n)$. Extracting connected components avoids this problem. This approach assumes that the representation of the connection components contained in set $C$ is $Y$ and that the point $p(\mathrm{~m}, \mathrm{n})$ in $Y$ is known. Then, all elements of $Y$ are generated using the Equation expressed iteratively as follows:

$$
Y_{k}=\left(\mathrm{Y}_{k-1} \oplus D\right) \cap C \quad k=1,2,3, \ldots
$$

In this paper, connected components were extracted by the 8 connected neighboring pixels. Connected component sets with sizes of length $=[\min (C), \max (C)]$ were segmented by different dimensions. To avoid unnecessary calculation, connected components were divided by the 3-dimensional algorithm at most.

$$
\operatorname{dim}=\left\{\begin{array}{rr}
1 & \min (\text { length })<\min (C) \leq \text { length } / 3 \\
2 & \text { length } / 3<\min (C) \leq 2 \min (\text { length }) / 3 \\
3 & 2 \min (\text { length }) / 3<\min (C) \leq \max (\text { length })
\end{array}\right.
$$

The food $F_{\mathrm{n}}^{L}$ obtained by the SSA is a 1-3-dimensional solution, based on the $F_{\mathrm{n}}^{L}$ segmentation image shown in Figure 5.






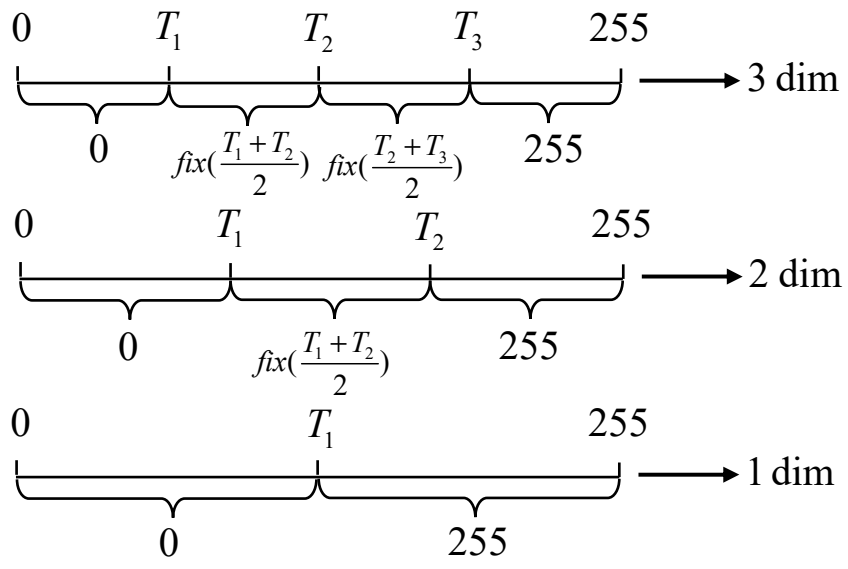

Figure 5. Multidimensional threshold segmentation schematic.

\subsection{Extraction of the Candidate MA Sets}

The main flow of this part is shown in Figure 6.

\subsubsection{MSA-K Algorithm}

The MSA-K algorithm is a quickly convergent version of the clustering algorithm proposed by Liwei at the 39th CCC International Conference. The steps of the MSA-K algorithm are as follows [36]:

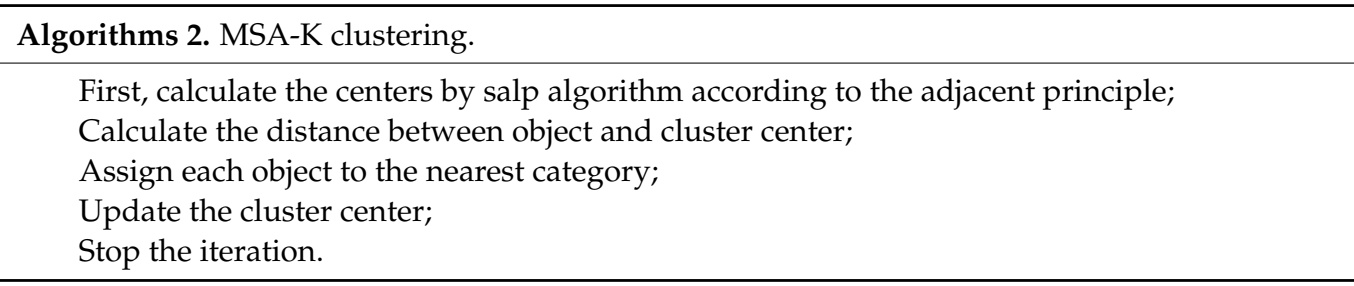

\subsubsection{Multifeature Clustering}

This section mainly concerns locating lesions in binary images obtained by CSSA segmentation. A candidate lesion set was obtained by MSA-K clustering, including the minimum length-width ratio of connected components bounding box $(T)$; the connected component, including the area of the connected component $(P)$, the farthest distance within the connected component $(L)$ and the radius-to-diameter ratio $(R D)$. The window of Radon transforms was then clustered to get the final candidate lesion set.

The blood vessels of binary images obtained by CSSA were easier to eliminate. More accurate candidate lesion sets were extracted by MSA-K clustering of $T, P, L$ and $R D$.

$T, P, L$ and $R D$ are defined as follows:

$$
\begin{gathered}
T=C / K \\
P=\int(x, y) \mathrm{d} x \\
L=\sqrt{2 \mu_{20}+\mu_{02}+\sqrt{\left(\mu_{20}+\mu_{02}\right)^{2}+4 \mu_{11}^{2}}} \\
R D=\frac{4 \pi P}{n^{2}}
\end{gathered}
$$




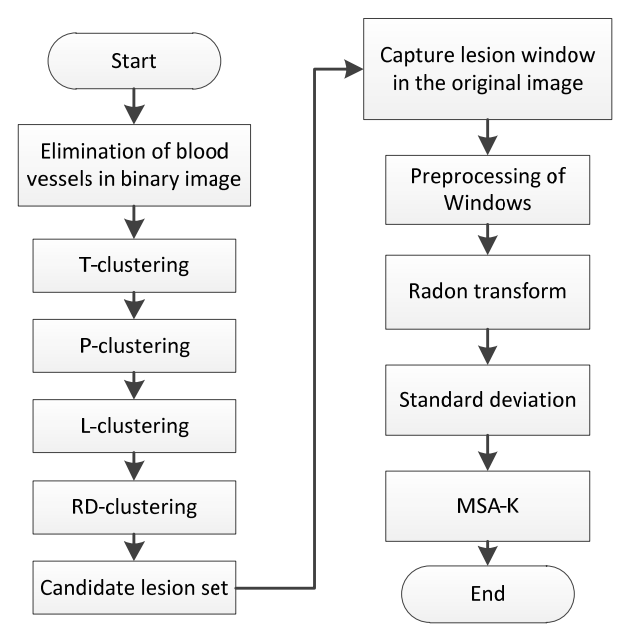

Figure 6. Localization of lesions.

\subsection{The Location of $M A s$}

A $50 \times 50$ template was used to capture MA windows from the original image. Make an inner circle of the window with the pixel outside the circle being 0 . This operation is to reduce the interference of the edge pixels in the subsequent multiangle Radon transform.

Radon transform is a mapping that projects an image in the direction of angle $\theta$. The transformation is as follows [37]:

$$
R_{\theta}=\iint R(\mathrm{x}, \mathrm{y}) \delta(\mathrm{s}-\mathrm{x} \cos \theta-\mathrm{y} \sin \theta) \mathrm{d} \mathrm{xdy}
$$

Figure 7 contains 4 typical lesions and the corresponding Radon transformation. In particular, (a) is a window that includes only an MA; the MA in (b) is near the blood vessel; and (c) and (d) are severed blood vessels wrongly identified as MAs.

As shown in Figure 7, it was found that the MA corresponded to a uniform and central light band in the Radon varying-frequency band. Therefore, the detection of MAs was achieved by the clustering of the standard deviations of the central Radon band.

In order to verify this point of view, the 3D graphs of a center circle and a diagonal line after Radon transformation are shown in Figure 8. As shown in Figure 8, the Radon transform of the circle was evenly distributed, and the height of the spine was close to unity. The window containing the straight line was mapped to the Radon domain through 18 angles $\left(0^{\circ}, 10^{\circ}, \ldots, 180^{\circ}\right)$. As shown in Figure $8 \mathrm{~b}$, the spine region was unevenly distributed and had mutations. This phenomenon was consistent with the theorem that the line integral of the same angle as the line is the largest. 


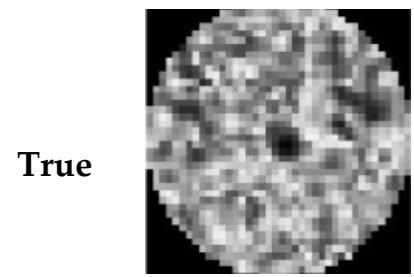

(a)

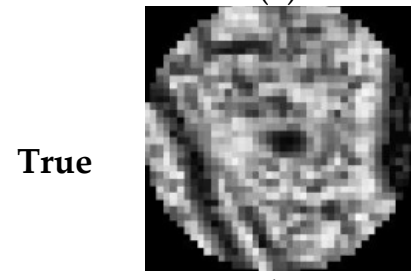

(b)

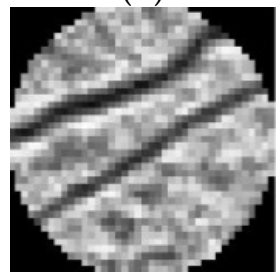

(c)



(d)



Radon (a)

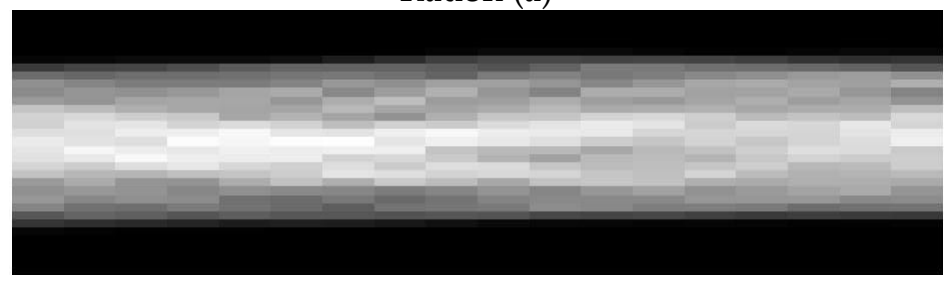

Radon (b)

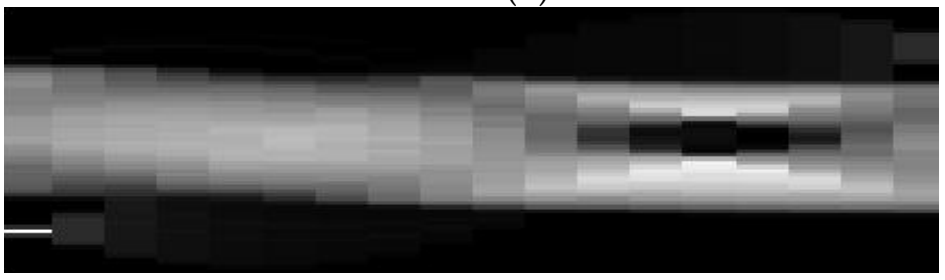

Radon (c)

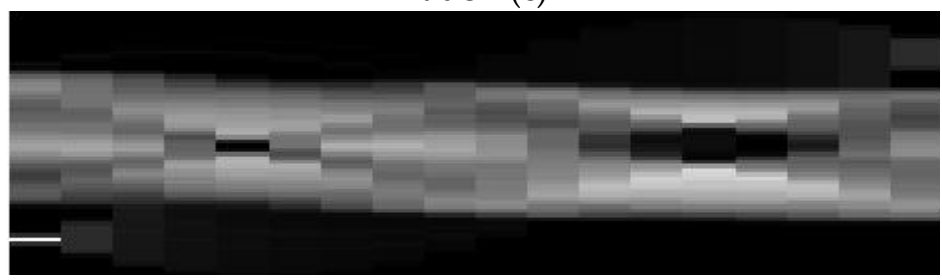

Radon (d)

Figure 7. Candidate lesion windows and Radon transforms. It contains 4 typical lesions and the corresponding Radon transformation. In particular, (a) is a window that includes only an MA; the MA in (b) is near the blood vessel; and (c,d) are severed blood vessels wrongly identified as MAs.

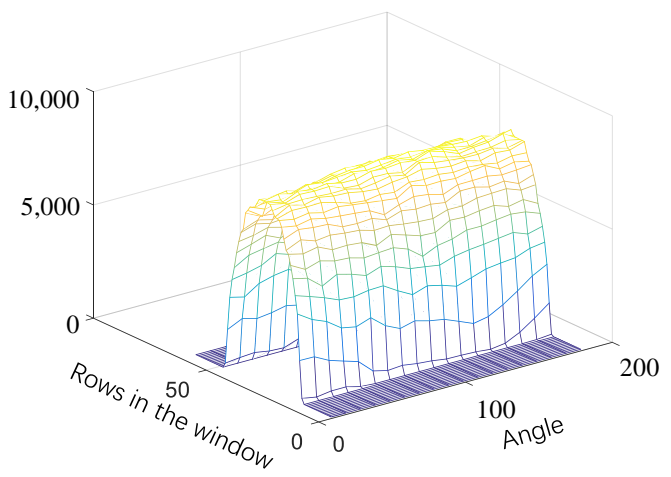

(a)



(b)

Figure 8. Eighteen-angle Radon transform 3D diagram: (a) is the Radon transform of a circle; (b) isthe Radon transform of an image containing a diagonal line. 
In order to prove the feasibility of the experiment, the process was reproduced for 20 MA windows and 20 noise windows. The results are shown in Figure 9. In Figure 9, the boundary between the standard deviations of true and false MAs is clear.

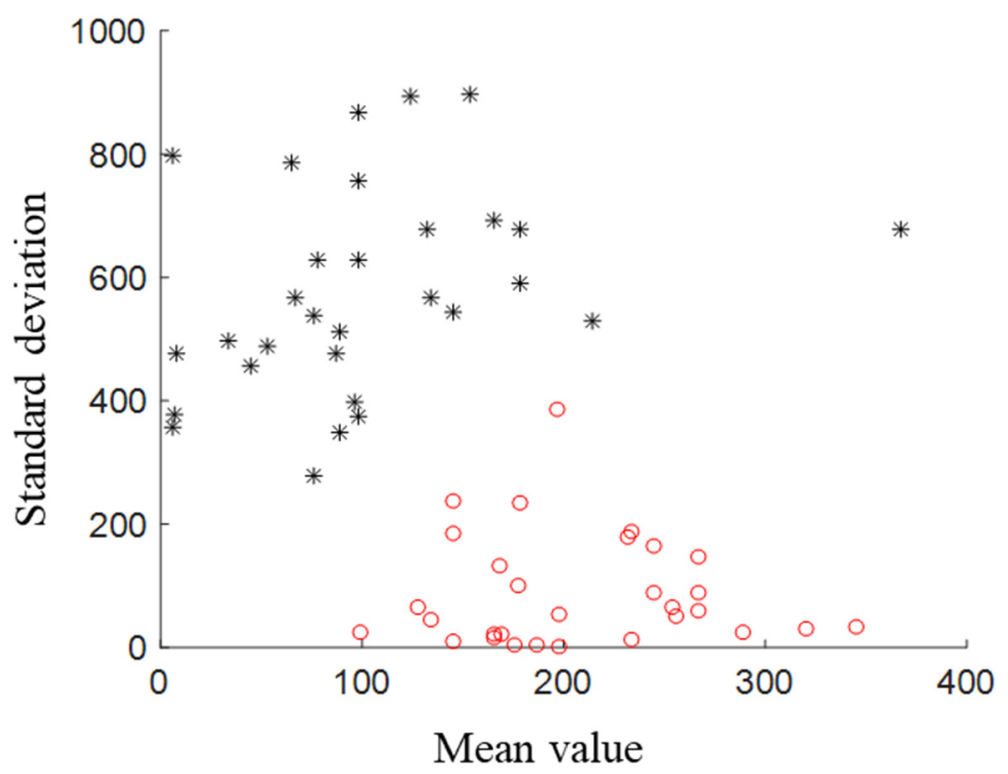

Figure 9. The standard deviation distribution of 18-angle Radon transformation, where o represents true MA and * represents false MA.

\section{Results}

\subsection{Evaluation Parameters and Data Analysis}

Peak signal-to-noise ratio (PSNR) was the basic criterion to evaluate the quality of the images [38]:

$$
\operatorname{PSNR}(\mathrm{M}, \mathrm{N})=10 \log _{10}\left(\mathrm{MAX}_{I}^{2} / \mathrm{MSE}(\mathrm{M}, \mathrm{N})\right)
$$

In order to evaluate whether there was damage in the structure of the segmented image, the SSIM parameter was selected as an evaluation criterion in this paper [39].

For images $\mathrm{X}$ and $\mathrm{Y}$, the parameter SSIM is defined as follows:

$$
\operatorname{SSIM}(\mathrm{M}, \mathrm{N})=l(\mathrm{M}, \mathrm{N}) c(\mathrm{M}, \mathrm{N}) s(\mathrm{M}, \mathrm{N})
$$

FSIM is a series of improvements on the basis of SSIM. The evaluation criterion considers that different pixels in an image have different weights. For example, the pixels at the edge of an object are more important than other pixels in defining the structure of the image [40]. FSIM is defined as follows:

$$
F S I M=\frac{\sum_{X \in \Omega} S_{o}(\mathrm{X}) P C_{p}(\mathrm{X})}{\sum_{X \in \Omega} P C_{p}(\mathrm{X})}
$$

\subsection{Simulation Results}

Table 1 is a comparison of SSIM, PSNR and FSIM in the different algorithms. In order to make the correlation among the data clearer, Table 1 was translated into Figure 10.

Table 2 shows the fitness values of the different algorithms. In order to make the data more intuitive, Table 2 can be interpreted with a radar chart. Figure 11 is the radar chart of SSIM, PSNR and FSIM.

The chord graph is mainly used to show the relationships between multiple objects; the connection between two points on the circle shows the relationship between the two corresponding objects, and the width of the line indicates the degree of the proportional relationship between the two objects. The same color in Figure 11a represents the fitness 
value of a particular algorithm for different dimensions. The same color in Figure 11b represents the fitness value of the same dimension for different algorithms.

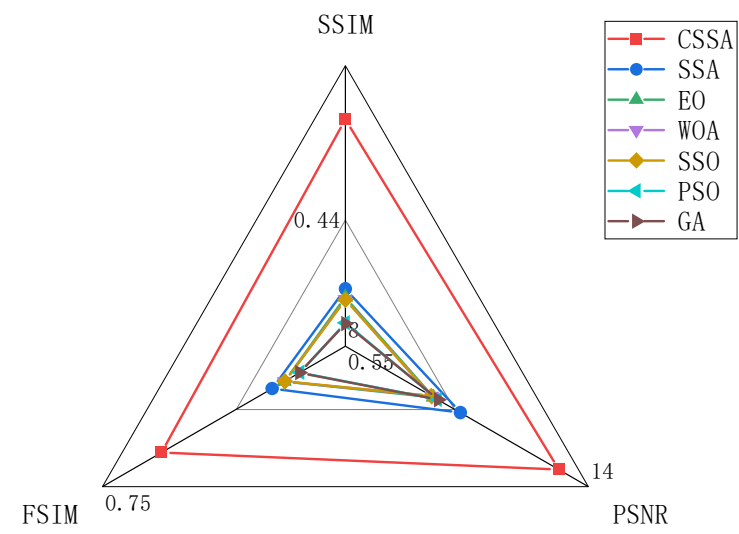

Figure 10. The radar chart of SSIM, PSNR and FSIM.

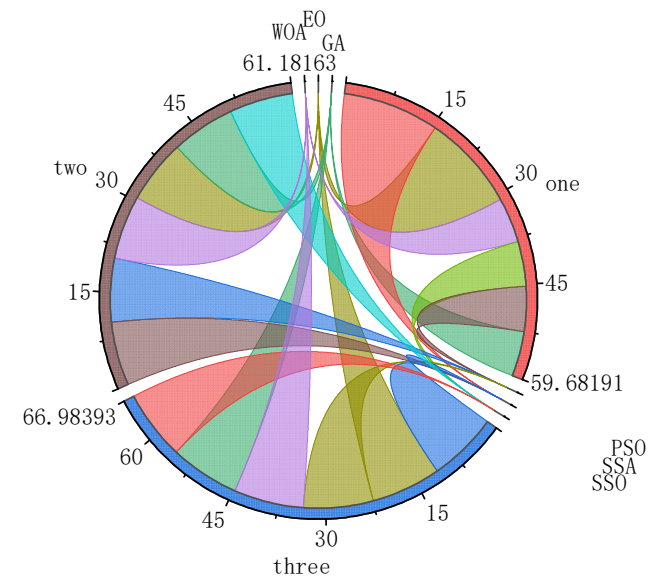

(a)



(b)

Figure 11. The chord graph of Table 2. (a) is the chord diagram of each algorithm for three dimensions, and (b) is the chord diagram of each dimension for the algorithms.

Table 1. Comparison of SSIM, PSNR and FSIM in the different algorithms.

\begin{tabular}{ccccccccc}
\hline Parameter & Image & CSSA & SSA & EO & WOA & SSO & PSO & GA \\
\hline \multirow{5}{*}{ SSIM } & (a) & 0.5038 & 0.4472 & 0.4372 & 0.4372 & 0.4372 & 0.4362 & 0.4372 \\
& (b) & 0.4921 & 0.3642 & 0.3638 & 0.3595 & 0.3595 & 0.3595 & 0.3595 \\
& (c) & 0.5714 & 0.4763 & 0.4685 & 0.4673 & 0.4653 & 0.4653 & 0.4653 \\
& (d) & 0.3770 & 0.2834 & 0.2740 & 0.2705 & 0.2705 & 0.2705 & 0.2672 \\
& (e) & 0.6993 & 0.4285 & 0.4194 & 0.4194 & 0.4194 & 0.4095 & 0.4070 \\
& (f) & 0.4256 & 0.3467 & 0.3467 & 0.3467 & 0.3467 & 0.2594 & 0.2594 \\
\hline \multirow{5}{*}{ PSNR } & (a) & 11.660 & 10.321 & 10.288 & 10.288 & 10.2880 & 10.247 & 10.288 \\
& (b) & 13.013 & 9.542 & 8.151 & 8.002 & 8.002 & 8.002 & 8.002 \\
& (c) & 14.915 & 14.343 & 14.343 & 14.277 & 14.214 & 14.214 & 14.214 \\
& (d) & 13.188 & 6.138 & 4.128 & 4.061 & 4.061 & 4.061 & 4.000 \\
& (e) & 13.360 & 12.995 & 12.995 & 12.995 & 12.995 & 12.861 & 12.951 \\
& (f) & 13.464 & 11.677 & 11.162 & 11.162 & 11.162 & 12.259 & 12.259 \\
\hline \multirow{3}{*}{ FSIM } & (a) & 0.6047 & 0.5821 & 0.5821 & 0.5821 & 0.5821 & 0.5802 & 0.5821 \\
& (b) & 0.6406 & 0.5121 & 0.5052 & 0.5018 & 0.5018 & 0.5018 & 0.5018 \\
& (c) & 0.7241 & 0.7119 & 0.7119 & 0.7111 & 0.7094 & 0.7094 & 0.7094 \\
& (d) & 0.7599 & 0.5518 & 0.5399 & 0.5428 & 0.5428 & 0.5428 & 0.5432 \\
& (e) & 0.7485 & 0.6561 & 0.6315 & 0.6315 & 0.6315 & 0.5949 & 0.5949 \\
& (f) & 0.7321 & 0.6472 & 0.6315 & 0.6315 & 0.6315 & 0.5949 & 0.5949 \\
\hline
\end{tabular}


Table 2. Comparison of the fitness values of each algorithm (shows 4 figures after the decimal point).

\begin{tabular}{|c|c|c|c|c|c|c|c|c|}
\hline Image & Dim & CSSA & SSA & EO & WOA & SSO & PSO & GA \\
\hline \multirow{3}{*}{ (a) } & 1 & \multirow{3}{*}{$\mathrm{N} / \mathrm{A}$} & 2.2290 & 2.2290 & 2.2290 & 2.2290 & 2.2290 & 2.2290 \\
\hline & 2 & & 3.0843 & 3.0843 & 3.0843 & 3.0829 & 3.0843 & 3.0804 \\
\hline & 3 & & 3.3790 & 3.3789 & 3.3789 & 3.3690 & 3.3790 & 3.3673 \\
\hline \multirow{3}{*}{ (b) } & 1 & \multirow{3}{*}{$\mathrm{N} / \mathrm{A}$} & 1.1624 & 1.1624 & 1.1623 & 1.1623 & 1.1623 & 1.1623 \\
\hline & 2 & & 1.7351 & 1.7351 & 1.7351 & 1.7303 & 1.7351 & 1.7344 \\
\hline & 3 & & 1.8900 & 1.8900 & 1.8900 & 1.8829 & 1.8900 & 1.8833 \\
\hline \multirow{3}{*}{ (c) } & 1 & \multirow{3}{*}{$\mathrm{N} / \mathrm{A}$} & 9.1490 & 9.1490 & 0.9144 & 0.9144 & 0.9144 & 0.9144 \\
\hline & 2 & & 1.2219 & 1.2219 & 1.2218 & 1.2179 & 1.2218 & 1.2213 \\
\hline & 3 & & 1.3520 & 1.3520 & 1.3512 & 1.3482 & 1.3518 & 1.3387 \\
\hline \multirow{3}{*}{ (d) } & 1 & \multirow{3}{*}{$\mathrm{N} / \mathrm{A}$} & 0.7069 & 0.7069 & 0.7068 & 0.7068 & 0.7068 & 0.7068 \\
\hline & 2 & & 1.0987 & 1.0987 & 1.0987 & 1.0978 & 1.0987 & 1.0982 \\
\hline & 3 & & 1.1963 & 1.1963 & 1.1963 & 1.1938 & 1.1963 & 1.1953 \\
\hline \multirow{3}{*}{ (e) } & 1 & \multirow{3}{*}{$\mathrm{N} / \mathrm{A}$} & 1.0396 & 1.0396 & 1.0396 & 1.0396 & 1.0396 & 1.0396 \\
\hline & 2 & & 1.4652 & 1.4642 & 1.4651 & 1.4642 & 1.4652 & 1.4651 \\
\hline & 3 & & 1.6046 & 1.6046 & 1.6036 & 1.6045 & 1.6045 & 1.6038 \\
\hline \multirow{3}{*}{ (f) } & 1 & \multirow{3}{*}{$\mathrm{N} / \mathrm{A}$} & 1.1496 & 1.1496 & 1.1496 & 1.1496 & 1.1496 & 1.1496 \\
\hline & 2 & & 1.5949 & 1.5949 & 1.5949 & 1.5938 & 1.5949 & 1.5943 \\
\hline & 3 & & 1.7547 & 1.7547 & 1.7546 & 1.7405 & 1.7547 & 1.7511 \\
\hline
\end{tabular}

Since the CSSA needs to calculate the threshold value of each connected component, it is meaningless to compare the CPU time (mean run time) of the CSSA with those of other global threshold algorithms. The computing speed of the CSSA can be evaluated by comparing the CPU time of the SSA with those of other algorithms. In this paper, the run time of all algorithms was the average run time (1-3D) of the same standard (same nonrepresentative code, iterated 1000 times) and segmentation standard on the same equipment (CPU: AMD A8-5550 M; graphics card: AMD Radeon HD 8670 M + AMD Radeon HD 8550 G; RAM: RY: 4 GB). The results are shown in Table 3.

In conclusion, the standard deviation of the window by Radon transformation can be used as the standard for dividing MA. The final result is shown in Figure 12.

(a)
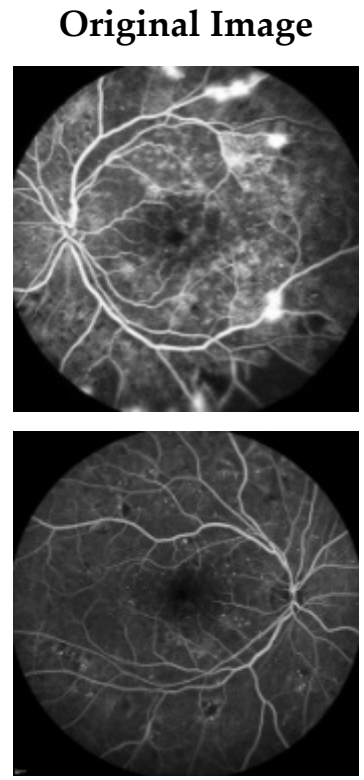

Image by CSSA


MA Detection
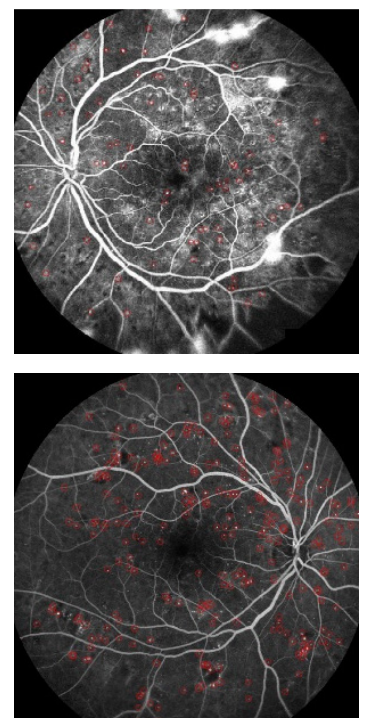

Figure 12. Cont. 
(c)

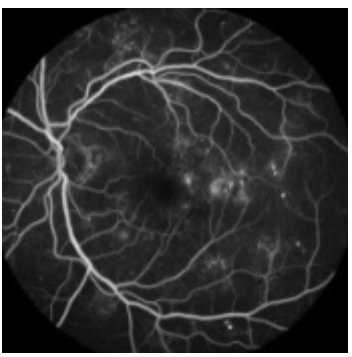

(d)



(e)

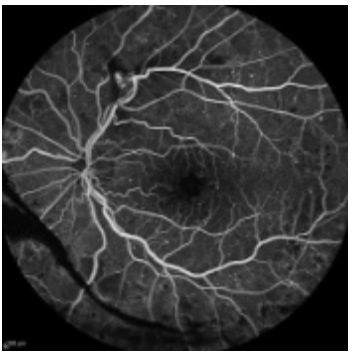

$(\mathbf{f})$



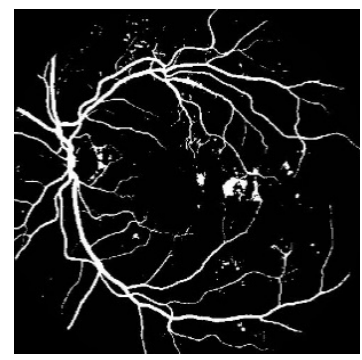
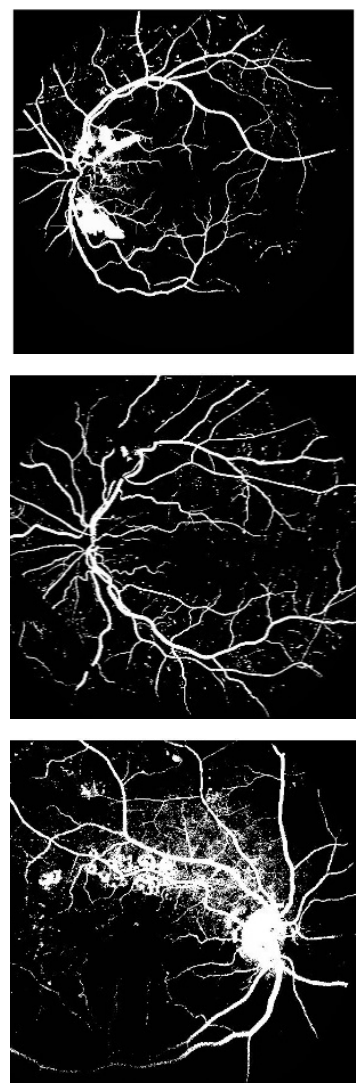
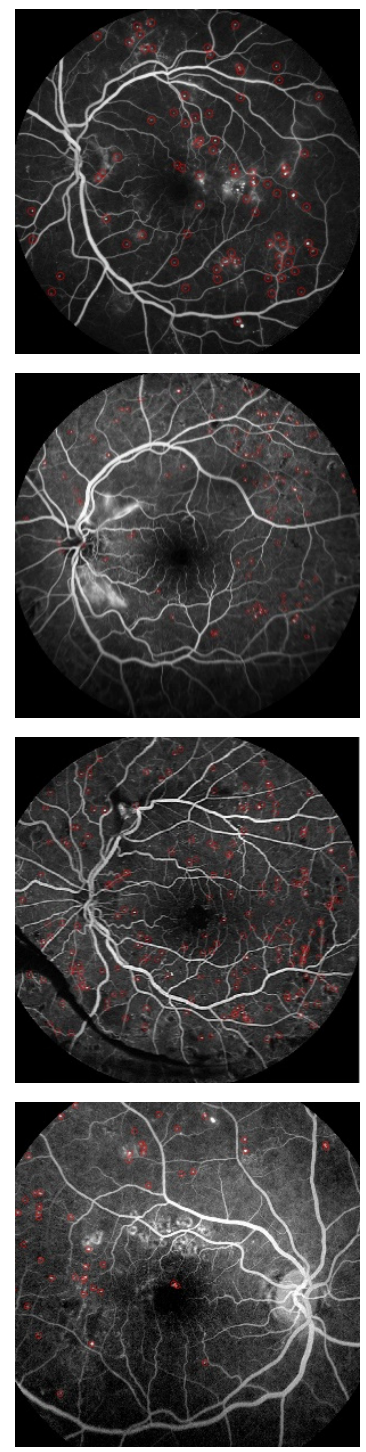

Figure 12. Locations of lesions in DR images. Left, the original image; middle, the binary image obtained by the method of this paper; right, the location of the final lesion.

Table 3. The mean run time of algorithm (shows 4 figures after the decimal point; unit: minutes).

\begin{tabular}{cccccccc}
\hline Name & CSSA & SSA & EO & WOA & SSO & PSO & GA \\
\hline $\begin{array}{c}\text { Average } \\
\begin{array}{c}\text { CPU } \\
\text { time }\end{array}\end{array}$ & N/A & 0.2559 & 0.9824 & 0.3459 & 0.8632 & 0.6538 & 0.6982 \\
\hline
\end{tabular}

Table 4 shows the accuracy of the algorithm in this paper and several other algorithms with the highest accuracy in locating fundus lesions.

Table 4. Accuracy of lesion localization.

\begin{tabular}{ccccccc}
\hline Algorithm & (a) & (b) & (c) & (d) & (e) & (f) \\
\hline CSSA & 90.62 & 92.47 & 94.78 & 93.32 & 95.02 & 93.25 \\
[29] & 89.72 & 90.30 & 85.43 & 87.32 & 94.03 & 92.08 \\
[31] & 87.04 & 85.32 & 87.03 & 89.06 & 90.68 & 89.72 \\
\hline
\end{tabular}




\section{Discussion}

The above experimental results showed that compared with the other two classic MA detection methods, the average accuracy of asymmetric MA detection in this paper was higher. The method of asymmetric MA detection in this article consisted of two parts. The first part was the improvement of the SSA to obtain a binary image containing MAs. This improvement solves the difficulty in medical image recognition that MAs are treated as noise to be canceled in preprocessing and contributes to subsequent MA detection. The second part was clustering the binary image based on T, P, L and RD to extract the candidate MA set. Then MA set was mapped to the Radon frequency domain based on the morphological characteristics of MAs. Finally, MSA-K clustering Radon (MA) was used to detect MAs. Therefore, the experimental verification also contained two parts.

The first was the evaluation of the binary image obtained by the improved CSSA. In order to objectively and quantitatively verify the performance of the binary image obtained by this method, the method was compared with the classical algorithms. The CSSA is an improved multidimensional algorithm based on the SSA for obtaining binary images that contain MAs. Therefore, the performance of the CSSA can be represented by the SSA. Figure 10 visualizes the excellence of the binary images obtained by CSSA in terms of SSIM, PSNR and FSIM.

In this part of the experiment, a radar chart and a chord chart were used to clearly interpret the experimental data. By observing the proportion of the target in Figure 11a, it was found that the fitness value of the three-dimensional algorithm was the best at $66.98 \%$. Correspondingly, the SSA had the highest proportion of overall dimensions in Figure 11b. This means that the performance of the binary images obtained by CSSA was comprehensive optimization.

The other part of the experiment was a comparative experiment between the method developed in this paper and several classic MA detection methods for accuracy. The results showed that the accuracy of the asymmetric MA detection algorithm in this paper was the highest and that the average accuracy could reach $93.24 \%$.

Limitations: compared with similar swarm optimization algorithms, the SSA has faster convergence speed and relatively excellent exploration and exploitation ability, but the limitation of the SSA is that $c_{1}=2 e^{-\left(\frac{4 L}{T}\right)^{2}}$ is related to the preset number of iterations $\mathrm{L}$, which makes the minimum number of iterations for the algorithm to obtain the optimal solution proportional to L. This means that, in order to make the algorithm converge faster, it is necessary to determine the complexity of the research problem in advance and determine the maximum number of iterations $\mathrm{L}$. This necessitates adding one more step: problem preprocessing before solving problems by SSA. This will be addressed in further work. In addition, the detection of MA in color fundus images will be studied in the future.

\section{Conclusions}

Since the number of MA pixels has an extremely low global scale, inherent image preprocessing regards MAs as noise to be canceled. Therefore, asymmetric MA detection has always been a difficulty in fundus image processing. In this paper, we solved this problem in three steps. First, we merged multiple connected components and the SSA to obtain a binary image containing MAs. Second, this binary image was multifeature clustered using the MSA-K algorithm to obtain the set of candidate MAs. Finally, asymmetric MA detection was achieved by clustering the standard deviation of the center band in the $\operatorname{Radon}\left(\right.$ window $\left._{j}\right)$.

The semisupervised learning method based on the SSA proposed in this paper achieved high accuracy in MA detection; the average accuracy of this method could reach $93.24 \%$. 
Author Contributions: Conceptualization, X.W. and L.D.; methodology, L.D.; software, X.W.; validation, X.W., J.X. and L.D.; formal analysis, X.W. and L.D.; investigation, L.D. and J.X.; resources, J.X.; writing—original draft preparation, X.W.; writing—review and editing, X.W.; visualization, L.D.; supervision, L.D.; project administration, J.X.; funding acquisition, L.D. All authors have read and agreed to the published version of the manuscript.

Funding: This research was funded by the National Science Foundation for Young Scientists of China, grant number (Grant No.61806060), 2019-2021, "Research of Diabetic Retinal Image Analysis Algorithms Based on Fractional Order Differential and the Deep Learning", and Natural Science Foundation of Heilongjiang Province (LH2019F024), China, 2019-2021, "Research on The Key Technology of Defect Detection of Wind Turbine Blade Based on Unmanned Aerial Vehicle-Taken Images".

Institutional Review Board Statement: Not applicable.

Informed Consent Statement: Not applicable.

Data Availability Statement: Data is contained within the article.

Conflicts of Interest: The authors declare no conflict of interest.

\section{References}

1. Scott, I.U.; Jackson, G.R.; Quillen, D.A.; Klein, R.; Liao, J.; Gardner, T.W. Effect of doxycycline vs placebo on retinal function and diabetic retinopathy progression in mild to moderate nonproliferative diabetic retinopathy: A randomized proof-of-concept clinical trial. JAMA Ophthalmol. 2014, 132, 1137-1142. [CrossRef]

2. Prentasic, P.; Loncaric, S.; Vatavuk, Z.; Bencic, G.; Tadic, R. Diabetic retinopathy image database (DRiDB): A new database for diabetic retinopathy screening programs research. In Proceedings of the International Symposium on Image \& Signal Processing \& Analysis, Trieste, Italy, 4-6 September 2013.

3. Yau, J.W.; Rogers, S.L.; Kawasaki, R.; Lamoureux, E.L.; Kowalski, J.W.; Bek, T.; Chen, S.-J.; Dekker, J.M.; Fletcher, A.; Grauslund, J.; et al. Global prevalence and major risk factors of diabetic retinopathy. Diabetes Care 2012, 35, 556-564. [CrossRef] [PubMed]

4. Ogurtsova, K.; Fernandes, J.D.R.; Huang, Y.; Linnenkamp, U.; Guariguata, L.; Cho, N.; Cavan, D.; Shaw, J.; Makaroff, L. IDF Diabetes Atlas: Global estimates for the prevalence of diabetes for 2015 and 2040. Diabetes Res. Clin. Pr. 2017, 128, 40-50. [CrossRef] [PubMed]

5. Yuanyuan, B.; Yujie, F.; Jie, M.; Huigang, W.; Pinghua, Z.; Juan, Y.; Yongfeng, D.; Qiaoyue, L.; Xinwen, Y.; Minglan, G.; et al. Serum glycated albumin may be a good biomarker for predicting type 2 diabetes: A retrospective cohort study based on the China National Diabetes and Metabolic Disorders survey (CNDMDS). Diabetes Metab. Res. Rev. 2021, 2021, e3477. [CrossRef]

6. Wu, B.; Zhu, W.; Shi, F.; Zhu, S.; Chen, X. Automatic detection of microaneurysms in retinal fundus images. Comput. Med. Imaging Graph. 2017, 55, 106-112. [CrossRef]

7. Dai, B.; $\mathrm{Wu}, \mathrm{X}$; $\mathrm{Bu}, \mathrm{W}$. Retinal microaneurysms detection using gradient vector analysis and class imbalance classification. PLoS ONE 2016, 11, e0161556. [CrossRef]

8. Mohanbabu, A.; Deepika, S.; Vidhya, R.J.; Santhana, M.; Sruthilakshmi, R. Retinal microaneurysms detection using local convergence index features. Int. J. Innov. Technol. Explor. Eng. (IJITEE) 2019, 8, 7-11.

9. Bernardes, R.; Nunes, S.; Pereira, I.; Torrent, T.; Rosa, A.; Coelho, D.; Cunha-Vaz, J. Computer-assisted microaneurysm turnover in the early stages of diabetic retinopathy. Ophthalmologica 2009, 223, 284-291. [CrossRef]

10. Tavakoli, M.; Shahri, R.P.; Pourreza, H.; Mehdizadeh, A.; Banaee, T.; Toosi, M.H.B. A complementary method for automated detection of microaneurysms in fluorescein angiography fundus images to assess diabetic retinopathy. Pattern Recognit. 2013, 46, 2740-2753. [CrossRef]

11. Qiao, S.; Pang, S.; Luo, G.; Pan, S.; Wang, X.; Wang, M.; Zhai, X.; Chen, T. Automatic detection of cardiac chambers using an attention-based YOLOv4 framework from four-chamber view of fetal echocardiography. arXiv 2020, arXiv:2011.13096.

12. Agúndez, J.A.; Emma, S. Perspective on the use of genomic biomarkers in the clinical setting. Pharmacogenomics 2014, 15, 419-422. [CrossRef] [PubMed]

13. Quan, Q.; Qingzhan, Z.; Manqing, L. Application of threshold segmentation in early diagnosis of diabetic retinopathy. J. Qingdao Univ. (Nat. Sci.) 2011, 24, 39-43.

14. Hassan, G.; Hassanien, A.E. Retinal fundus vasculature multilevel segmentation using whale optimization algorithm. Signal Image Video Process. 2017, 12, 263-270. [CrossRef]

15. Al-Rawi, M.; Karajeh, H. Genetic algorithm matched filter optimization for automated detection of blood vessels from digital retinal images. Comput. Methods Programs Biomed. 2007, 87, 248-253. [CrossRef] [PubMed]

16. Arnay, R.; Fumero, F.; Sigut, J. Ant Colony Optimization-based method for optic cup segmentation in retinal images. Appl. Soft Comput. 2017, 52, 409-417. [CrossRef]

17. Bian, J.H.; Ren, X.T. Flower pollination algorithm based on elitist reservation mechanism. J. Harbin Univ. Commer. Nat. Sci. Ed. 2016, 32, 3 . 
18. Hu, H.; Kantardzic, M.; Sethi, T.S. No Free Lunch Theorem for concept drift detection in streaming data classification: A review. Wiley Interdiscip. Rev. Data Min. Knowl. Discov. 2020, 10, e1327. [CrossRef]

19. Mirjalili, S.; Gandomi, A.H.; Mirjalili, S.Z.; Saremi, S.; Faris, H.; Mirjalili, S.M. Salp Swarm Algorithm: A bio-inspired optimizer for engineering design problems. Adv. Eng. Softw. 2017, 114, 163-191. [CrossRef]

20. Bao, L.; Lv, X.; Yao, J. Water Extraction in SAR Images Using Features Analysis and Dual-Threshold Graph Cut Model. Remote Sens. 2021, 13, 3465. [CrossRef]

21. Bogiatzis, A.; Papadopoulos, B. Global Image Thresholding Adaptive Neuro-Fuzzy Inference System Trained with Fuzzy Inclusion and Entropy Measures. Symmetry 2019, 11, 286. [CrossRef]

22. Majumdar, A.; Debnath, T.; Biswas, A.; Sood, S.K.; Baishnab, K.L. An Energy Efficient e-Healthcare Framework Supported by

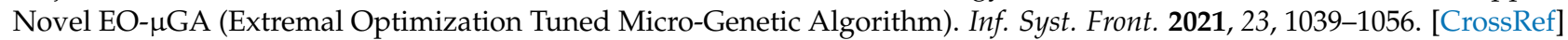

23. Nasiri, J.; Khiyabani, F.M. A whale optimization algorithm (WOA) approach for clustering. Cogent Math. Stat. $2018,5,1483565$. [CrossRef]

24. James, J.Q.; Li, V.O. A social spider algorithm for global optimization. Appl. Soft Comput. 2015, 30, 614-627.

25. Jagtap, H.; Bewoor, A.; Kumar, R.; Ahmadi, M.H.; Lorenzini, G. Markov-based performance evaluation and availability optimization of the boiler-furnace system in coal-fired thermal power plant using PSO. Energy Rep. 2020, 6, 1124-1134. [CrossRef]

26. Rarità, L.; Stamova, I.; Tomasiello, S. Numerical schemes and genetic algorithms for the optimal control of a continuous model of supply chains. Appl. Math. Comput. 2020, 388, 125464. [CrossRef]

27. Asiri, N.; Hussain, M.; Adel, F.A.; Aboalsamh, H. A deep learning-based unified framework for red lesions detection on retinal fundus images. arXiv 2021, arXiv:2109.05021.

28. Wan, C.; Chen, Y.; Li, H.; Zheng, B.; Chen, N.; Yang, W.; Wang, C.; Li, Y. EAD-Net: A novel lesion segmentation method in diabetic retinopathy using neural networks. Dis. Markers 2021, 2021, 6482665. [CrossRef]

29. Quellec, G.; Lamard, M.; Josselin, P.M.; Cazuguel, G.; Cochener, B.; Roux, C. Optimal wavelet transform for the detection of microaneurysms in retina photographs. IEEE Trans. Med. Imaging 2008, 27, 1230-1241. [CrossRef]

30. Hervella, Á.S.; Rouco, J.; Novo, J.; Ortega, M. Retinal microaneurysms detection using adversarial pre-training with unlabeled multimodal images. Inf. Fusion 2021, 79, 146-161. [CrossRef]

31. Saleh, M.D.; Eswaran, C. An automated decision-support system for non-proliferative diabetic retinopathy disease based on MAs and HAs detection. Comput. Methods Programs Biomed. 2012, 108, 186-196. [CrossRef] [PubMed]

32. Jin, K.; Pan, X.; You, K.; Wu, J.; Liu, Z.; Cao, J.; Lou, L.; Xu, Y.; Su, Z.; Yao, K.; et al. Automatic detection of non-perfusion areas in diabetic macular edema from fundus fluorescein angiography for decision making using deep learning. Sci. Rep. 2020, 10, 1-7. [CrossRef] [PubMed]

33. Chang, Z.; Cao, J.; Zhang, Y. A novel image segmentation approach for wood plate surface defect classification through convex optimization. J. For. Res. 2018, 29, 1789-1795. [CrossRef]

34. Otsu, N. A Threshold Selection Method from Gray-Level Histograms. IEEE Trans. Syst. Man. Cybern. 1979, 9, 62-66. [CrossRef]

35. Qi, Q.; Zhao, Q.Z.; Deng, H.T. Location of microaneurysms on diabetic retinopathy images based on extraction of connection components. In Proceedings of the 2011 International Conference on Computer and Management (CAMAN), Wuhan, China, 19-21 May 2011. [CrossRef]

36. Deng, L.; Wang, X.; Ai, L. A new fracture image segmentation method based on MSA-k clustering algorithm. In Proceedings of the 2020 39th Chinese Control Conference (CCC), Kunming, China, 22-24 May 2020; pp. 1002-1006.

37. Zhang, L.; Wang, Y.; Zhang, D. Research on multiple-image encryption mechanism based on Radon transform and ghost imaging. Opt. Commun. 2021, 504, 127494. [CrossRef]

38. Setiadi, D.R.I.M. PSNR vs SSIM: Imperceptibility quality assessment for image steganography. Multimed. Tools Appl. 2021, 80, 8423-8444. [CrossRef]

39. Wang, S.; Jia, H.; Peng, X. Modified salp swarm algorithm based multilevel thresholding for color image segmentation. Math. Biosci. Eng. 2020, 17, 700-724. [CrossRef] [PubMed]

40. Zhang, L.; Zhang, L.; Mou, X.; Zhang, D. FSIM: A feature similarity index for image quality assessment. IEEE Trans. Image Process. 2011, 20, 2378-2386. [CrossRef] [PubMed] 